\title{
Implications of new clinical practice guidance on familial hypercholesterolaemia for Australian general practitioners
}

CPD

Tom Brett, Jan Radford, Clare Heal, Charlotte Mary Hespe, Jacquie Garton-Smith, Andrew Kirke, Dick C Chan, Jing Pang, Gerald F Watts

\section{Background}

Familial hypercholesterolaemia $(\mathrm{FH})$ is a monogenic lipid disorder that may be overlooked in the diagnostic process.

\section{Objective}

The aim of this article is to review the key areas for identification and management of $\mathrm{FH}$ that affect Australian general practitioners (GPs).

\section{Discussion}

Recent consensus advice on the care of patients with $\mathrm{FH}$ in Australia provides an opportunity for GPs to increase their awareness and skills in diagnosing and managing $\mathrm{FH}$. New Medicare Benefits Schedule items for genetic testing and Pharmaceutical Benefits Scheme listing for the use of proprotein convertase subtilisin/kexin 9 (PCSK9) inhibitors offer GPs additional supports to improve the care of patients with $\mathrm{FH}$. A shared-care approach between GPs and non-GP specialists with expertise in multiple disciplines offers the best option to facilitate genetic testing and management of index cases and affected family relatives. Implementation of this guidance in the primary care setting remains an ongoing challenge and needs to be embraced as a high priority.

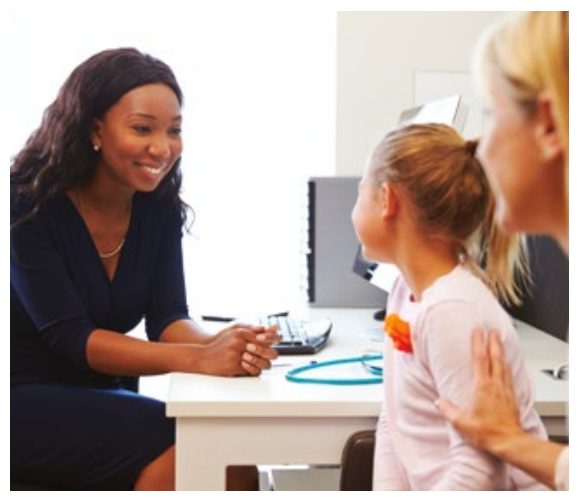

RECENT CONSENSUS ADVICE on integrated guidance to enhance the care of patients with familial hypercholesterolaemia (FH) in Australia provides a timely reminder and opportunity for general practitioners (GPs) to increase their awareness and skill in diagnosing and managing the condition. ${ }^{1}$

With $88 \%$ of Australians presenting to their GPs annually, ${ }^{2}$ GPs are well placed to play a more active part. Currently, fewer than $10 \%$ of Australia's 100,000 patients with FH are diagnosed, with most failing to achieve optimum management and therapeutic targets. ${ }^{3}$

The hereditary nature of $\mathrm{FH}$, with its high cholesterol burden present from birth, ${ }^{3}$ causes premature atherosclerotic cardiovascular disease (ASCVD), principally coronary artery disease (CAD), and death if left untreated. ${ }^{4,5} \mathrm{FH}$ is caused by a defect in the low-density lipoprotein (LDL) receptor pathway, and it affects one in 250-300 Australians. ${ }^{6,7}$ Among first-degree relatives, $50 \%$ are affected because of its autosomal dominant, monogenic inheritance and high penetrance. ${ }^{3}$

General practice is central to the continuity of care and advocacy for all patients with $\mathrm{FH}$ and their families. This involves a role in screening, diagnosis, shared care with specialists, overseeing cholesterol-lowering medications and multimorbidities, as well as applying context-specific models of care for $\mathrm{FH} .^{1,8}$

This article focuses on key recommendations for the identification and management of heterozygous FH derived from the recently published integrated guidance, ${ }^{1}$ with particular reference to GPs. The aim of this article is to help translate this updated guidance into everyday health policy and practice, and facilitate the provision of high-quality healthcare for patients with $\mathrm{FH}$ and their families in the primary care setting.

\section{New genetic tests and impact on clinical care}

The Australian Government has introduced new pathology services to assist with detection of heritable mutations predisposing to $\mathrm{FH}$ : 
Medicare Benefits Schedule (MBS) Item 73352 for index cases and Item 73353 for cascade testing of close relatives. ${ }^{9}$ Item 73352 requires non-GP specialist authorisation, but Item 73353 can be requested by the patient's GP.

These new tests provide diagnostic precision, offering GPs added incentive to increase diagnosis in the young when treatment can be most effective. ${ }^{4,9}$ The level of evidence is high, and the class of recommendation is strong for using genetic testing to confirm the diagnosis. This is especially important when cascade testing is planned. ${ }^{1,10,11}$

Since more than $20 \%$ of probable or definite FH may not have a detectable mutation, ${ }^{11} \mathrm{FH}$ should not be totally excluded if a pathogenic, or likely pathogenic, gene variant is not detected. ${ }^{1,4,12}$

A shared-care approach between GP and non-GP specialists (ie lipidologist, cardiologist, pediatrician $)^{13}$ can facilitate genetic testing of index cases. Pre- and post-test counselling should be an integral part of the process. ${ }^{1}$ Once an index case is genetically proven, GPs can offer cascade testing among first- and second-degree relatives, arrange appropriate counselling and collaborate with non-GP specialists in risk stratification and treatment. ${ }^{8,12}$ As discussed later, screening requires appropriate GP education, screening tools and skill training in the care of patients with FH.

Figure 1 summarises genetic screening and management of an individual at high risk of $\mathrm{FH}$.

Risk notification of family members requires special skills to overcome potential barriers, such as privacy laws, poor communication in families, poor health literacy, geographical location and psychological issues. ${ }^{8,11}$ Coordination of the overall process remains a significant challenge, especially from current inadequate general practice infrastructure..$^{1,8,14}$

\section{Diagnosis in adults}

Awareness of FH among Australian GPs Despite the high prevalence of $\mathrm{FH}$ and an ever-increasing number of research publications, awareness about $\mathrm{FH}$ in the general community and among many health professionals remains suboptimal. ${ }^{3}$ The concept of increased cholesterol burden from birth in affected individuals is not well understood. In addition, the hereditary 'familial' component of FH, with its associated premature ASCVD, tends to remain unrecognised and untreated. ${ }^{3}$

Young people have most to gain from early diagnosis and appropriate treatment. ${ }^{4,15,16}$ Apart from lifestyle interventions, most patients will need lifelong cholesterol-lowering medications, especially statins. Ezetimibe and proprotein convertase subtilisin/kexin 9 (PCSK9) inhibitors may be needed for cases that are more difficult to control. . $^{3,417}$

Failure to diagnose $\mathrm{FH}$ before middle age results in up to $50 \%$ of untreated men developing myocardial infarction or angina by the age of 50 years, and $30 \%$ of untreated women similarly affected by the age of 60 years. ${ }^{5}$ Early recognition and treatment produces significant reductions in cardiac morbidity and mortality. ${ }^{15,16}$ Strict adherence to treatment allows affected individuals to live a normal lifespan. ${ }^{3}$

\section{History-taking}

The importance of effective historytaking in clinical medicine is one of the fundamental tenets of diagnostic evaluation. However, poor-quality medical information in practice records may affect the diagnosis. GPs will often be aware of patients and families with a history of premature coronary events attending their practice. Linking such histories, combined with a lipid profile showing total cholesterol $>7.5 \mathrm{mmol} / \mathrm{L}$ or LDL cholesterol (LDL-C) $>5.0 \mathrm{mmol} / \mathrm{L}$, should alert the GP to the possibility of FH. Potential secondary causes of hypercholesterolaemia (diabetes, hypothyroidism, steroid use, renal and liver disease) should be excluded before $\mathrm{FH}$ is further assessed. ${ }^{3}$

Personal or family history of premature CAD and markedly elevated cholesterol levels, both central to the Dutch Lipid Clinic Network Criteria (DLCNC) score, are the chief drivers for establishing a phenotypic FH diagnosis. Assessment of non-cholesterol risk factors, genetic testing and cardiovascular imaging can improve risk re-stratification and optimise treatment. ${ }^{3}$

\section{Electronic health record screening}

FH meets all the criteria for worthwhile disease screening. ${ }^{8}$ Various approaches have been suggested - including universal, opportunistic and selective screening but all require effective coordination for maximum benefit. ${ }^{8,10}$

Reverse child-parent screening, ${ }^{18}$ targeting premature myocardial infarcts in coronary care units and screening electronic health records (EHRs) in general practice all offer promise..$^{8,19,20}$ Laboratory report alerts also help with index case detection..$^{1,8,20}$

\section{Purposeful clinical examination}

Once FH diagnosis is considered, clinical examination should focus on physical stigmata including premature corneal arcus and tendon xanthomata. ${ }^{3}$ Periorbital xanthelasmata are suggestive of hypercholesterolaemia, and their presence should alert the GP to the potential of underlying FH. Additional training for GPs in pattern recognition and early consideration of FH among close family members could help increase awareness of key hereditary features.

The DLCNC score combines personal and family history of premature CAD, ${ }^{1,3,8}$ elevated LDL-C levels and physical stigmata to establish phenotypic $\mathrm{FH}$ diagnosis in high-risk patients. In a genetic analysis of $\mathrm{FH}$ in Australia, 70\% of patients with a definite $\mathrm{FH}$ diagnosis were found to have an $\mathrm{FH}$-causing mutation, but only $29 \%$ of patients with probable $\mathrm{FH}$ and $11 \%$ of patients with possible $\mathrm{FH}$ were mutation positive. ${ }^{21}$

\section{Diagnosis in children and adolescents}

The cumulative LDL-C burden in patients with $\mathrm{FH}$ starts from birth and progressively increases over their lifetimes. ${ }^{4}$ However, most children with $\mathrm{FH}$ are asymptomatic and have no clinical signs. Hence, the current largest gap is the early detection of children with $\mathrm{FH} .{ }^{3}$

Early detection is an important component of providing optimum 


\section{General practice clinics}

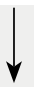

Patient clinically reviewed and scored according to DLCNC

Identification of high risk of FH

- DLCNC score

- $L D L-C \geq 6.5 \mathrm{mmol} / \mathrm{L}$ in the absence of secondary causes

- LDL-C 5.0-6.6 mmol/L with signs of premature or accelerated atherogenesis

\section{GP standard} care for phenotypic FH Care plans 721/723

Mutation
negative

\section{GP referral to a specialist to request $\mathrm{FH}$ genetic testing MBS Item $\mathbf{7 3 3 5 2}$}

(if not previously tested)

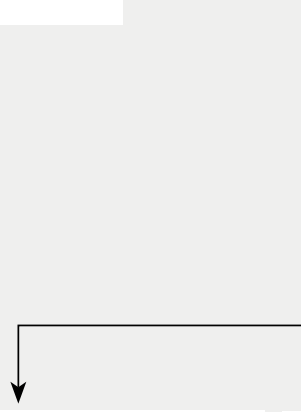

Low complexity

- Absence of CVD

- Absence of risk factors for CVD

- Reached LDL-C goal with a statin

\section{Intermediate complexity}

- Stable CVD and risk factors

- Close to LDL-C goal with statin

- Low-grade statin intolerance

- HeFH aged $<18$ years

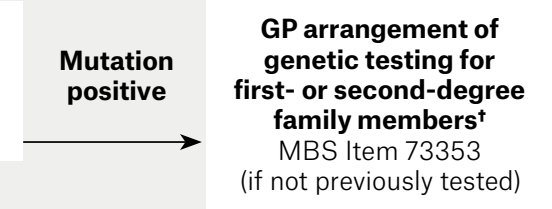

Mutation positive

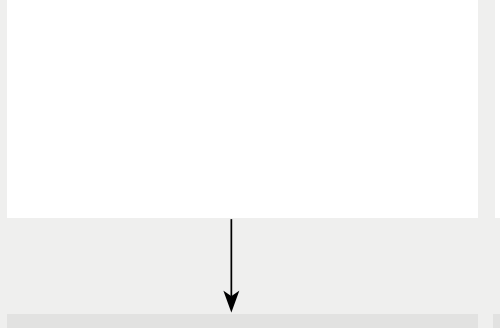

Primary care

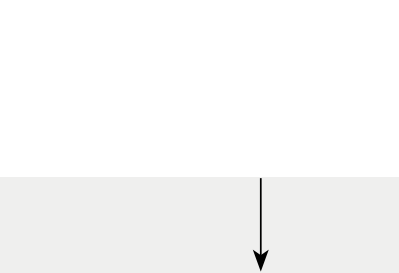

Shared care

\section{High complexity}

- Numerous uncontrolled CVD risk

factors

- Symptomatic CVD

- Failure to reach LDL-C goal despite statin/ezetimibe

- High-level statin intolerance

- Children, pregnancy, need for PCSK9 inhibitors or LDL-C apheresis

- $\mathrm{HeFH}>18$ years or $\mathrm{HoFH}$

- Help with cascade screening

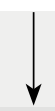

Tertiary care

Figure 1. Process for genetic screening and management of an individual at high risk of familial hypercholesterolaemia ${ }^{*}$ Refer to Sturm AC, Knowles JW, Gidding SS, et al, Clinical genetic testing for familial hypercholesterolemia: JACC Scientific Expert Panel, J Am Coll Cardiol 2018;72(6):662-80. doi: 10.1016/j.jacc.2018.05.044.

${ }^{\dagger}$ Genetic cascade testing may be undertaken by a general practitioner with skills in the care of patients and families with $\mathrm{FH}$, under the guidance of an appropriate specialist. Consent is obtained from the index case to contact family. The process of risk notification of family members should be consistent with relevant local legislation and institutional guidelines. Risk notification may be indirect (providing a family letter for the notifier to pass to relatives) or direct (clinical service writes to relatives); pre-and post-test genetic counselling should be offered to all at-risk family members.'

CVD, cardiovascular disease; DLCNC, Dutch Lipid Network Criteria; FH, familial hypercholesterolaemia; GP, general practitioner; HeFH, heterozygous familial hypercholesterolaemia; HoHF, homozygous familial hypercholesterolaemia; LDL-C, low-density lipoprotein cholesterol; PCSK9, proprotein convertase subtilisin/kexin 9 
management, most likely statins, from the age of $8-10$ years. ${ }^{1}$ Use of statins in children will need parental consent with adequate explanation of its benefit and possible side effects. The benefits outweigh any risks for most children.

The LDL-C level drives the phenotypic diagnosis, but genetic cascade testing offers the definitive diagnosis. ${ }^{4,11}$ Risk stratification helps to facilitate more rational and precise treatment. ${ }^{8}$

The DLCNC score is not suitable for use in children and adolescents. ${ }^{3}$ Children with an LDL-C $\geq 5 \mathrm{mmol} / \mathrm{L}$ have a high risk of $\mathrm{FH}$; for children with premature CAD in close relatives and/or baseline high cholesterol in one parent, an LDL-C $\geq 4 \mathrm{mmol} / \mathrm{L}$ is indicative of high $\mathrm{FH}$ risk. ${ }^{4}$

The current guidelines do not recommend specific cut-off LDL-C levels for parental hypercholesterolaemia. ${ }^{1,4}$ The value of non-invasive cardiovascular imaging tools, such as the carotid intima-media thickness test to improve the early diagnosis of $\mathrm{FH}$ in children, needs further evaluation.

\section{Management}

\section{Adults}

There is compelling evidence from extensive clinical trial, registry and genetic data for patients with $\mathrm{FH}$ to be actively treated with cholesterol-lowering medication, diet and lifestyle modification from an early age. ${ }^{8,12}$ Other risk factors should also be addressed. Shared doctorpatient decision making helps adherence to therapy. ${ }^{22}$

Cumulative evidence supports a $50 \%$ reduction in LDL-C, plus low absolute targets depending on primary or secondary prevention settings. ${ }^{8,23,24}$ Such targets are rarely achieved, with evidence from a Danish study showing less than 50\% adherence to treatment even with an established FH diagnosis, ${ }^{25}$ consistent with a recent $\mathrm{FH}$ registry report in Australia. ${ }^{26}$ Preliminary evidence from an Australian primary care study shows a similar pattern. ${ }^{27}$

To reach very low LDL-C levels, patients may require sequential treatment with a high-potency statin, ezetimibe and PCSK9 inhibitor, ${ }^{8,22,23}$ the latter now listed on the Pharmaceutical Benefits Scheme (PBS) requiring initiation by a non-GP specialist. ${ }^{28}$ Follow-up consultations can be managed in primary care for patients with low-complexity FH. Greater awareness by Australian GPs would help optimise shared-care responsibility with specialists in multiple disciplines for patients at greatest risk. ${ }^{8}$

FH treatment from childhood is supported by good-quality observational studies. ${ }^{4,8}$ Modest, sustained reductions in LDL-C starting early in life can have a major effect in preventing future premature mortality due to ASCVD. ${ }^{4,8,10,18}$ A healthy lifestyle, while important, does not sufficiently lower LDL-C.

A low-potency statin, with or without ezetimibe, may be required from the age of 10 years. ${ }^{4,8,10,15,29,30}$ LDL-C targets do not need to be as low as for adults, ${ }^{8,16}$ but medication safety should be continually monitored. .,

Family-based clinics with paediatric specialist involvement that address therapy adherence are essential for enhancing care. ${ }^{1,8}$ If uncertainty exists and GPs feel reluctant to initiate or continue statin treatment, support from a paediatric specialist with expertise in lipidology should be sought. ${ }^{1,3}$

Box 1 summarises recommendations and therapeutic targets, based on moderate levels of evidence and class of recommendation, for managing FH. ${ }^{1,24,26,29,30}$

\section{Pregnancy}

Statins and other systemically absorbed cholesterol-lowering medications should be ceased three months before planned conception as well as during pregnancy and while breastfeeding. ${ }^{1}$ All women of childbearing age with $\mathrm{FH}$ should be offered pre-pregnancy counselling prior to starting treatment with statins. Appropriate contraceptive advice that is reinforced at least annually helps minimise

Box 1. Low-density lipoprotein cholesterol treatment targets and recommendations for the management of familial hypercholesterolaemia

\section{Adults}

- Commencement of statin treatment should be considered once a diagnosis of FH is confirmed.

- LDL-C targets can be divided as follows:

- LDL-C $<2.5 \mathrm{mmol} / \mathrm{L}$ (absence of ASCVD or other major ASCVD risk factors) 1,20,21

- LDL-C $<1.8 \mathrm{mmol} / \mathrm{L}$ (imaging evidence of ASCVD alone or other major ASCVD risk factors $)^{1,20,21}$

- LDL-C $<1.4 \mathrm{mmol} / \mathrm{L}$ (presence of clinical ASCVD). ${ }^{1,20,21}$

\section{Children}

- Commencement of statin treatment should be considered by age 8-10 years irrespective of sex. $1.4,6,8,13,20,27$

- For children with FH aged 8-10 years on a suitable diet, an LDL-C treatment target $<4.0 \mathrm{mmol} / \mathrm{L}$ or a $30-40 \%$ reduction in LDL-C may be acceptable. . $1,6,13,27^{2}$

- In children aged >10 years who are maintained on a suitable diet, an LDL-C treatment target $<3.5 \mathrm{mmol} / \mathrm{L}$ or a $50 \%$ reduction in LDL-C may be acceptable..$^{1,4,1,13,27}$

- Statin therapy with or without ezetimibe in addition to diet/exercise should be used to reach the above targets. ${ }^{14,6,8,13,27}$

- Statins licensed for use in children aged 8-10 years (pravastatin, fluvastatin, simvastatin) should be used; ezetimibe is licensed from the age of 10 years and should be used accordingly., ${ }^{1,2}$

- Atorvastatin and rosuvastatin use should be considered according to the clinical indications and shared decision making. ${ }^{1,6}$

- PCSK9 inhibitors are not generally recommended in children but may be considered in heterozygous $\mathrm{FH}$ according to clinical indications and shared decision making. Further clinical trials on long-term safety are required.

ASCVD, atherosclerotic cardiovascular disease; FH, familial hypercholesterolaemia; LDL-C, low-density lipoprotein cholesterol; PCSK9, proprotein convertase subtilisin/kexin 9 
cardiovascular risk. GPs should always seek specialist support for patients with $\mathrm{FH}$ who are considering pregnancy. ${ }^{3}$

The importance of early diagnosis and treatment of FH in girls should never be underestimated. ${ }^{31}$ Pregnancy and lactation can result in the loss of effective years of statin treatment due to childbearing. ${ }^{31}$ Adherence to statin treatment can be difficult, especially in young people. The need for early and ongoing treatment at optimal doses should be stressed to improve future ASCVD outcomes. ${ }^{31}$ The cost-effectiveness of such an approach is increasingly recognised. ${ }^{32}$

\section{Conclusion}

This guidance is aligned with a recent international global call to action on $\mathrm{FH}^{33}$ The recommendations are designed to be incorporated into healthcare pathways that meet the needs of the Australian population. ${ }^{3,8}$ Several efforts have been made to improve care of patients with $\mathrm{FH}$ in primary care. These include increased recognition of phenotypic diagnosis of $\mathrm{FH}$, greater consideration of screening in children, increased awareness of the need to employ implementation science and practice to optimise health service delivery, and advocacy groups. The introduction of MBS items for genetic testing and PBS-supported use of PCSK9 inhibitors is likely to improve considerably the care of patients with $\mathrm{FH}$ in the future. ${ }^{9,12,28}$ Education and skill training for GPs, such as cascade screening, risk notification, and pre- and post-test genetic counselling, are paramount for implementing effective care in general practice.

The challenge we now face is how to implement this guidance into health policy and high-quality care. Implementation research and practice will need to be embraced as a high priority to increase the impact of this guidance on improving the care of all Australians who have, or are at risk of, $\mathrm{FH}^{33}$

\section{Key points}

- Recent guidelines support improved care of patients with $\mathrm{FH}$ in the primary care setting.
- GPs are ideally placed to play a proactive part in diagnosing $\mathrm{FH}$ and then co-managing these patients in conjunction with other specialists.

- New MBS and PBS items enable more precise diagnosis and treatment of $\mathrm{FH}$.

\section{Authors}

Tom Brett MA, MD, FRACGP, MRCGP, Professor and Director, General Practice and Primary Health Care Research, School of Medicine, University of Notre Dame, Fremantle, WA; General Practitioner, Mosman Park Medical Centre, Mosman Park, WA Jan Radford MBBS, MPsychMed, MEd, FRACGP, FARGP, GAID, AFANZAHPE, Associate Professor of General Practice, Launceston Clinical School, Tasmanian School of Medicine, University of Tasmania, Launceston, Tas; General Practitioner, West Tamar Health, Riverside, Tas; Provost, The Royal Australian College of General Practitioners Tasmanian Faculty, Hobart, Tas

Clare Heal MBChB, DRANZCOG, DipGUMed, FRACGP, MPHTM, PhD, Promotional Chair, Discipline of General Practice and Rural Medicine, Mackay Clinical School, James Cook University College of Medicine and Dentistry, Mackay, Qld

Charlotte Mary Hespe FRACGP, MBBS (Hons), DCH (Lon), Associate Professor and Head of General Practice and Primary Care Research, School of Medicine Sydney, University of Notre Dame, Sydney, NSW; General Practitioner, Glebe Family Medical Practice, Glebe, NSW

Jacquie Garton-Smith MBBS, FRACGP, Clinical Co-Lead, Cardiovascular Health Network, Western Australian Department of Health, East Perth, WA; Clinical Services, Royal Perth Hospital, Perth, WA Andrew Kirke MBBS, FRACGP, FACRRM, Head, Rural Clinical School of Western Australia, University of Western Australia, West Busselton, WA

Dick C Chan BSc, MPhil, PhD, FRCPath, Senior Research Fellow, School of Medicine, Faculty of Health and Medical Sciences, University of Western Australia, Crawley, WA; General Practice and Primary Health Care Research Unit, School of Medicine, University of Notre Dame, Fremantle, WA Jing Pang BSc, PhD, National Health and Medical Research Council Early Career Fellow, School of Medicine, Faculty of Health and Medical Sciences, University of Western Australia, Crawley, WA

Gerald F Watts DSc, PhD, MD, FRACP, FRCP, Winthrop Professor and Consultant Physician, School of Medicine, Faculty of Health and Medical Sciences, University of Western Australia, Crawley, WA; Lipid Disorders Clinic, Cardiometabolic Service, Departments of Cardiology and Internal Medicine, Royal Perth Hospital, Perth, WA

Competing interests: TB reports that Amgen supported travel and accommodation costs for attendance at FH Summit in Melbourne (February 2019), and provided honorarium for $\mathrm{FH}$ meeting in Dublin (September 2018). JGS reports employment with WA Primary Health Alliance. JR reports that Amgen supported travel and accommodation costs for attendance at FH Summit in Melbourne (February 2019).

Funding: The authors acknowledge current National Health Medical Research Council (NHMRC) Partnership Grant Funding (GNT11428830). TB, $\mathrm{CMH}, \mathrm{CH}$ and JGS report grant funding from Sanofi-Aventis (Study Number DIREGL07823). CMH reports an independent research grant from Amgen.
GFW reports research grants and personal fees from Arrowhead, Amgen, Sanofi, and Regeneron, and grants from Novartis and advisory board personal fees from Kowa and AstraZeneca, outside the submitted work.

Provenance and peer review: Not commissioned, externally peer reviewed.

\section{Correspondence to:}

tom.brett@nd.edu.au

\section{References}

1. Watts GF, Sullivan DR, Hare DL, et al. Integrated guidance for enhancing the care of familial hypercholesterolaemia in Australia. Heart Lung Circ 2021;30(3):324-49. doi: 10.1016/j. hlc.2020.09.943.

2. The Royal Australian College of General Practitioners. General practice: Health of the nation 2018. East Melbourne, Vic: RACGP, 2018. Available at www.racgp.org.au/download/ Documents/Publications/Health-of-the-Nation2018-Report.pdf [Accessed 10 December 2020].

3. Pang J, Sullivan DR, Brett T, Kostner KM, Hare DL, Watts GF. Familial hypercholesterolaemia in 2020: A leading tier 1 genomic application. Heart Lung Circ 2020;29(4):619-33. doi: 10.1016/j. hlc.2019.12.002.

4. Wiegman A, Gidding SS, Watts GF, et al. Familial hypercholesterolaemia in children and adolescents: Gaining decades of life by optimizing detection and treatment. Eur Heart J 2015;36(36):2425-37. doi: 10.1093/eurheartj/ ehv157.

5. Marks D, Thorogood M, Neil HA, Humphries SE. A review on the diagnosis, natural history, and treatment of familial hypercholesterolaemia. Atherosclerosis 2003;168(1):1-14. doi: 10.1016/ s0021-9150(02)00330-1.

6. Watts GF, Shaw JE, Pang J, Magliano DJ, Jennings GL, Carrington MJ. Prevalence and treatment of familial hypercholesterolaemia in Australian communities. Int J Cardiol 2015;185:69-71. doi: 10.1016/j.ijcard.2015.03.027.

7. Pang J, Martin AC, Mori TA, Beilin LJ, Watts GF. Prevalence of familial hypercholesterolemia in adolescents: Potential value of universal screening? J Pediatr 2016;170:315-16. doi: 10.1016/j.jpeds.2015.11.019.

8. Watts GF, Gidding SS, Mata P, et al. Familial hypercholesterolaemia: Evolving knowledge for designing adaptive models of care. Nat Rev Cardiol 2020;17(6):360-77. doi: 10.1038/s41569019-0325-8.

9. Australian Government Department of Health. Medicare Benefits Schedule 2020 - Item 73352. Canberra, ACT: MBS Online, 2020. Available at www9.health.gov.au/mbs/ fullDisplay.cfm?type=item\&q=73352\&qt=|temID [Accessed 10 December 2020].

10. Gidding SS, Champagne MA, de Ferranti SD, et al. The agenda for familial hypercholesterolemia: A scientific statement from the American Heart Association. Circulation 2015;132(22):2167-92. doi: 10.1161/CIR.0000000000000297.

11. Sturm AC, Knowles JW, Gidding SS, et al. Clinical genetic testing for familial hypercholesterolemia: JACC Scientific Expert Panel. J Am Coll Cardiol 2018;72(6):662-80. doi: 10.1016/j. jacc.2018.05.044.

12. Hooper AJ, Burnett JR, Bell DA, Watts GF. The present and the future of genetic testing in familial hypercholesterolemia: Opportunities and caveats. Curr Atheroscler Rep 2018;20(6):31. doi: 10.1007/ s11883-018-0731-0. 
13. Vickery AW, Bell D, Garton-Smith J, Kirke AB, Pang J, Watts GF. Optimising the detection and management of familial hypercholesterolaemia: Central role of primary care and its integration with specialist services. Heart Lung Circ 2014;23(12):1158-64. doi: 10.1016/j.hlc.2014.07.062.

14. Brett T, Qureshi N, Gidding S, Watts GF. Screening for familial hypercholesterolaemia in primary care: Time for general practice to play its part. Atherosclerosis 2018;277:399-406. doi: 10.1016/j.atherosclerosis.2018.08.019.

15. de Ferranti SD, Steinberger J, Ameduri R, et al. Cardiovascular risk reduction in highrisk pediatric patients: A scientific statement from the American Heart Association. Circulation 2019;139(13):e603-34. doi: 10.1161/ CIR.0000000000000618.

16. Luirink IK, Wiegman A, Kusters DM, et al. 20-year follow-up of statins in children with familial hypercholesterolemia. N Engl J Med 2019;381(16):1547-56. doi: 10.1056/ NEJMoa1816454.

17. Arnett DK, Blumenthal RS, Albert MA, et al. 2019 ACC/AHA guideline on the primary prevention of cardiovascular disease: A report of the American College of Cardiology/American Heart Association Task Force on Clinical Practice Guidelines. Circulation 2019;140(11):e596-646. doi: 10.1161/ CIR.0000000000000678.

18. Wald DS, Bestwick JP, Morris JK, Whyte K Jenkins L, Wald NJ. Child-parent familial hypercholesterolemia screening in primary care. N Engl J Med 2016;375(17):1628-37. doi: 10.1056/ NEJMoa1602777.

19. Troeung $L$, Arnold-Reed D, Chan She Ping-Delfos W, et al. A new electronic screening tool for identifying risk of familial hypercholesterolaemia in general practice. Heart 2016:102(11):855-61. doi: 10.1136/ heartjnl-2015-308824.

20. Kirke AB, Barbour RA, Burrows S, et al. Systematic detection of familial hypercholesterolaemia in primary health care: A community based prospective study of three methods. Heart Lung Circ 2015;24(3):250-56. doi: 10.1016/j. hlc.2014.09.011.

21. Hooper AJ, Nguyen LT, Burnett JR, et al. Genetic analysis of familial hypercholesterolaemia in Western Australia. Atherosclerosis 2012;224(2):430-34. doi: 10.1016/j. atherosclerosis.2012.07.030.

22. Kinnear FJ, Wainwright E, Perry Ret al. Enablers and barriers to treatment adherence in heterozygous familial hypercholesterolaemia: A qualitative evidence synthesis. BMJ Open 2019;9(7):e030290. doi: 10.1136/bmjopen-2019 030290.

23. Mach F, Baigent C, Catapano AL, et al. 2019 ESC/EAS guidelines for the management of dyslipidaemias: Lipid modification to reduce cardiovascular risk: The Task Force for the management of dyslipidaemias of the European Society of Cardiology (ESC) and European Atherosclerosis Society (EAS). Eur Heart J 2020:41(1):111-88. doi: 10.1093/eurheartj/ehz455.

24. Grundy SM, Stone NJ, Bailey AL, et al. 2018 AHA/ACC/AACVPR/AAPA/ABC/ACPM/ADA/ AGS/APhA/ASPC/NLA/PCNA guideline on the management of blood cholesterol: A report of the American College of Cardiology/American Heart Association Task Force on Clinical Practice Guidelines. Circulation 2019;139(25):e1082-143. doi: 10.1161/CIR.0000000000000625. Erratum in: Circulation 2019 Jun 18;139(25):e1182-86.

25. Benn M, Watts GF, Tybjaerg-Hansen A, Nordestgaard BG. Familial hypercholesterolemia in the Danish general population: Prevalence, coronary artery disease, and cholesterollowering medication. J Clin Endocrinol Metab 2012:97(11):3956-64. doi: 10.1210/jc.20121563. Erratum in: J Clin Endocrinol Metab 2014;99(12):4758-59.

26. Pang J, Sullivan DR, Hare DL, et al. Gaps in the care of familial hypercholesterolaemia in Australia: First report from the national registry. Heart Lung Circ 2021;30(3):372-79. doi: 10.1016/j. hlc.2020.07.012

27. Arnold-Reed DE, Brett $T$, Troeung $L$, et al. Detection and management of familial hypercholesterolaemia in primary care in Australia: Protocol for a pragmatic cluster intervention study with pre-post intervention comparisons. BMJ Open 2017;7(10):e017539. doi: 10.1136/bmjopen2017-017539.

28. Australian Government Department of Health. The Pharmaceutical Benefits Scheme schedule: Code no. 10958R. Canberra, ACT: Commonwealth of Australia, 2020. Available at www.pbs.gov.au/medicine/ item/10958r [Accessed 10 December 2020].

29. Raal FJ, Hovingh GK, Catapano AL. Familial hypercholesterolemia treatments: Guidelines and new therapies. Atherosclerosis 2018;277:483-92. doi: 10.1016/j.atherosclerosis.2018.06.859.

30. Ramaswami U, Humphries SE, Priestley-Barnham L, et al. Current management of children and young people with heterozygous familial hypercholesterolaemia - HEART UK statement of care. Atherosclerosis 2019;290:1-8. doi: 10.1016/j.atherosclerosis.2019.09.005.

31. Pang J, Chan DC, Watts GF. The knowns and unknowns of contemporary statin therapy for familial hypercholesterolemia. Curr Atheroscler Rep 2020;22(11):64. doi: 10.1007/s11883-02000884-2.

32. Ademi Z, Norman R, Pang J, et al. Health economic evaluation of screening and treating children with familial hypercholesterolemia early in life: Many happy returns on investment? Atherosclerosis 2020;304:1-8. doi: 10.1016/j. atherosclerosis.2020.05.007.

33. Representatives of the Global Familial Hypercholesterolemia Community, Wilemon KA, Patel J, et al. Reducing the clinical and public health burden of familial hypercholesterolemia: A global call to action. JAMA Cardiol 2020:5(2):217-29. doi: 10.1001/jamacardio.2019.5173. 